\title{
Geometry and Topology of Escape. II. Homotopic Lobe Dynamics
}

\author{
K. A. Mitchell \\ William \& Mary \\ J. P. Handley \\ William \& Mary \\ John B. Delos \\ William \& Mary, jbdelos@wm.edu \\ Stephen Knudson \\ William \& Mary
}

Follow this and additional works at: https://scholarworks.wm.edu/aspubs

Part of the Physics Commons

\section{Recommended Citation}

Mitchell, K. A.; Handley, J. P.; Delos, John B.; and Knudson, Stephen, Geometry and Topology of Escape. II. Homotopic Lobe Dynamics (2003). Chaos: An Interdisciplinary Journal of Nonlinear Science, 13(3), 892-902.

https://doi.org/10.1063/1.1598312

This Article is brought to you for free and open access by the Arts and Sciences at W\&M ScholarWorks. It has been accepted for inclusion in Arts \& Sciences Articles by an authorized administrator of W\&M ScholarWorks. For more information, please contact scholarworks@wm.edu. 


\title{
Geometry and topology of escape. II. Homotopic lobe dynamics
}

\author{
K. A. Mitchell, ${ }^{\text {a) }}$ J. P. Handley, and J. B. Delos ${ }^{\text {b) }}$ \\ Department of Physics, College of William and Mary, Williamsburg, Virginia 23187-8795 \\ S. K. Knudson \\ Department of Chemistry, College of William and Mary, Williamsburg, Virginia 23187-8795
}

(Received 1 October 2002; accepted 17 June 2003; published 15 August 2003)

\begin{abstract}
We continue our study of the fractal structure of escape-time plots for chaotic maps. In the preceding paper, we showed that the escape-time plot contains regular sequences of successive escape segments, called epistrophes, which converge geometrically upon each end point of every escape segment. In the present paper, we use topological techniques to: (1) show that there exists a minimal required set of escape segments within the escape-time plot; (2) develop an algorithm which computes this minimal set; (3) show that the minimal set eventually displays a recursive structure governed by an "Epistrophe Start Rule:" a new epistrophe is spawned $\Delta=D+1$ iterates after the segment to which it converges, where $D$ is the minimum delay time of the complex. (C) 2003 American Institute of Physics. [DOI: 10.1063/1.1598312]
\end{abstract}

Topological methods and symbolic dynamics have long been valuable tools for describing orbits of dynamical systems. For example, if a particle in the plane scatters from three fixed disks, labeled A, B, and C, its orbit can be characterized by a sequence of symbols, such as ... ABA*BCBCA ...., giving the sequence of collisions with the disks. The asterisk gives the location of the particle at the present time; as time goes by the asterisk takes one step to the right. In this paper, we describe a new kind of symbolic dynamics, in which the symbol sequence describes the structure of a curve in the plane. The relevant curve is not the trajectory of a particle, but rather an ensemble of initial points in phase space-the line of initial conditions. This line winds around "holes" in the plane in a manner described by the symbol sequence. The dynamical map applied to the line induces a map on the symbol sequence, which is more complicated than a simple shift. We use this symbolic dynamics to derive properties of the epistrophes introduced in the preceding paper. In particular, we use it to obtain a "minimal set of escape segments" and an "epistrophe start rule."

\section{INTRODUCTION}

As in the preceding paper $^{1}$ (Paper I), we study maps of the phase plane, having an unstable fixed point (an $X$ point) and an associated homoclinic tangle of stable and unstable manifolds (Fig. 1). The stable and unstable manifolds intersect transversely, bounding a region of phase space (called the "complex") from which a trajectory may or may not escape. We consider an initial distribution of points along a curve passing through the complex (the line of initial conditions). The escape-time plot is the number of iterates of the

${ }^{a)}$ Electronic mail: kevinm@physics.wm.edu

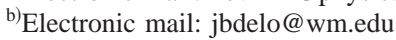

map required for a point to escape the complex, plotted as a function along the line of initial conditions (Fig. 2). For chaotic systems, such escape-time plots have a complicated set of singularities and structure at all levels of resolution. ${ }^{2-16}$ These fractal escape-time plots play a central role in a variety of classical decay and scattering problems; we have been particularly motivated by the ionization of atoms, especially hydrogen in parallel electric and magnetic fields.

The escape-time plot of a discrete map is divided into "escape segments," intervals over which the escape time is constant. In Paper I, we proved that there exist certain important sequences of consecutive escape segments, which we called epistrophes, at all levels of resolution. The epistrophes are characterized by the Epistrophe Theorem, whose core results are: (1) each end point of an escape segment spawns a new epistrophe which converges upon it; (2) in the limit $n_{i} \rightarrow \infty$, every epistrophe converges geometrically, with rate equal to the Liapunov factor $\alpha$ of the $X$ point; (3) the asymptotic tails of any two epistrophes differ by a simple scaling.

The focus of Paper I was the asymptotic behavior of epistrophes. In the present paper, we address how epistrophes begin. We use the topological structure of the homoclinic tangle and the line of initial conditions to show that there is a certain minimal set of escape segments. For this minimal set, we prove the Epistrophe Start Rule (Theorem 1 ), which says that after a sufficiently large time, each epistrophe begins some number of iterates $\Delta$ after the segment that spawned it (i.e., the segment upon which the epistrophe converges). The number $\Delta$ is the same for all epistrophes of a given map and is dependent on the topological structure of the tangle; explicitly, $\Delta=D+1$, where $D$ is the minimum delay time of the complex, that is, the minimum number of iterates a scattering trajectory spends inside the complex. The bulk of our effort is devoted to developing an algebraic algorithm for constructing the minimal set of escape segments for a general line of initial conditions. This algorithm allows us to compute the initial structure of the escape-time 


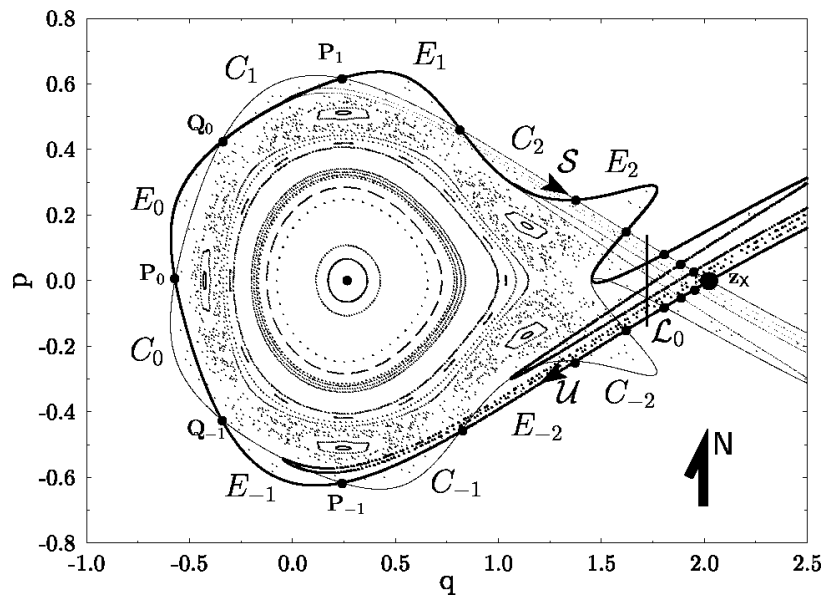

FIG. 1. A phase space portrait is shown for our saddle-center map, which possesses a homoclinic tangle. The line of initial conditions $\mathcal{L}_{0}$ coincides with $q=1.72$.

plot for iterates before the Epistrophe Start Rule sets in. The algorithm is then used to prove the Epistrophe Start Rule itself.

A critical aspect of this paper is our use of homotopy theory. We develop the necessary formalism in Sec. III, and prior knowledge of homotopy theory is not required. Homotopy theory provides an algebraic framework for describing the topological structure of curves in the phase plane. As we shall explain, the phase plane has a set of "holes" into which the line of initial conditions cannot pass. A symbol sequence can be used to describe how the line circumvents these holes. As the dynamics maps the line forward, there is an induced dynamics on the symbol sequence, representing a new kind

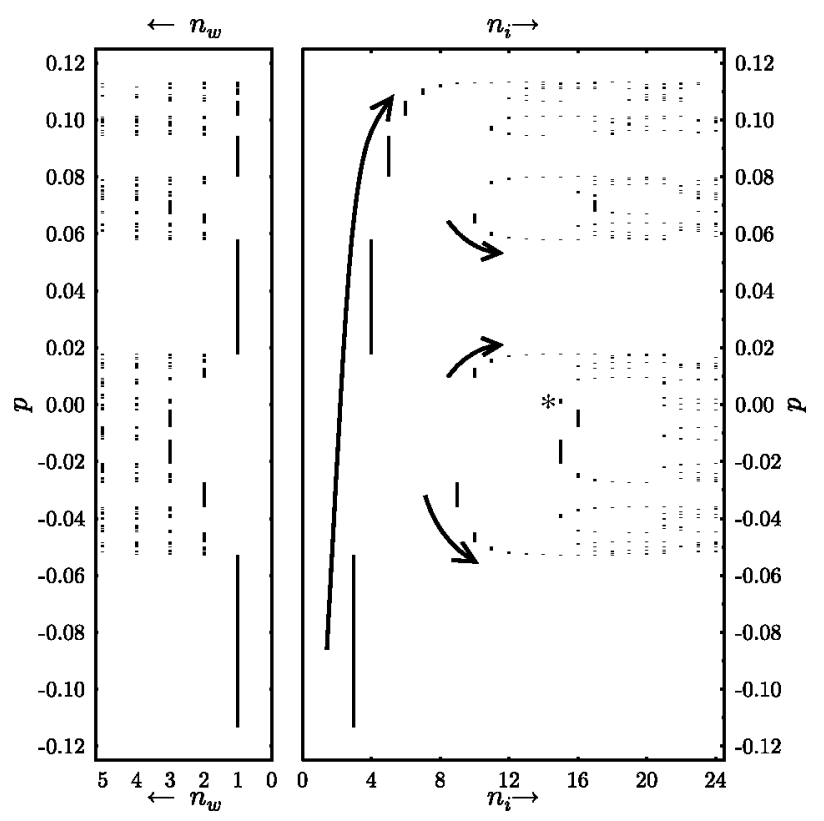

FIG. 2. Escape data $n_{i}$ and $n_{w}$ are plotted for the saddle-center map in Fig. 1. On the right, the number of iterates $n_{i}$ required to escape is plotted as a function of $p$ parametrizing the line of initial conditions $\mathcal{L}_{0}$. The escape segment marked by an asterisk at $n_{i}=15$ is the first numerically computed segment that is not in the minimal set; it is a strophe. On the left is plotted the winding number of the trajectory as it escapes to infinity. of symbolic dynamics which we call "homotopic lobe dynamics." From the symbol sequence, one can readily read off the structure of the minimal set of escape segments. Lines with different symbol sequences may have different minimal sets; however, at long enough times, these minimal sets always obey the Epistrophe Start Rule.

Some escape segments, such as that marked with an asterisk in Fig. 2, are not within the minimal set guaranteed by the topology. These segments are "surprises" which, within the present topological analysis, we cannot predict. Since they break the regular structure and since they often have no obvious connection with any of the epistrophes, we call them "strophes" as in Sec. III B of Paper I. Strophes interfere with the self-similar structure of the fractal and typically do not go away in the asymptotic limit, resulting in what we called "epistrophic self-similarity" in Sec. III C of Paper I. Despite the presence of these strophes, the minimal set often seems to accurately describe the early and intermediate time structure of the escape-time plot.

Patterns similar to our Epistrophe Start Rule have been seen in other work. In the numerical study of Tiyapan and Jaffé, ${ }^{14}$ epistrophes and the Epistrophe Start Rule are evident in the structure of the initial angle-final action plot (analogous to the escape-time plot). Similarly, Jung and co-workers ${ }^{9-11}$ used symbolic dynamics to construct a treediagram that gives a comparable description of a scattering system. In each case, the authors consider a specific line of initial conditions that is far outside of the complex and is topologically simple. Easton, ${ }^{17}$ followed by Rom-Kedar and others, ${ }^{18-20}$ showed that recursive patterns also apply to homoclinic intersections between the stable and unstable manifolds. Thus, it may not be surprising that comparable patterns should apply to the intersections between the stable manifold and an arbitrary line of initial conditions, at least at sufficiently large iterate. But at what iterate does this pattern set in, and what is the minimal set before it sets in? Algorithm 1 answers both these questions, as well as giving a simple proof of the Epistrophe Start Rule. An important observation is that the escape-time plot depends both on the topology of the tangle and on the topology of the line of initial conditions.

The paper is organized as follows. Section II motivates our study by presenting numerical computations on a particular saddle-center map with a chosen line of initial conditions. Section III is the theoretical heart of the paper, in which we formally develop homotopic lobe dynamics. Section IIID contains Algorithm 1 for computing the minimal set of escape segments. Section III F contains Theorem 1, which includes the Epistrophe Start Rule. In Sec. IV we apply our techniques to the escape-time plots for two representative lines of initial conditions. Conclusions are in Sec. V. Appendices A and B contain the proofs of Algorithm 1 and Theorem 1, respectively. Table I summarizes the notation in this article.

\section{NUMERICAL DATA FOR AN EXAMPLE SYSTEM}

As an example we study the map $\mathcal{M}$ defined by Eqs. (A1)-(A3) of Paper I using parameter values $\tau=1.5, f$ 
TABLE I. Notation summary.

\begin{tabular}{ll}
\hline \hline $\mathcal{M}$ & Dynamical map \\
$\mathbf{z}_{\mathbf{X}}$ & Unstable fixed point \\
$\mathcal{S}, \mathcal{U}$ & Tangled branches of the stable and unstable \\
& manifolds \\
$\mathbf{P}_{n}, \mathbf{Q}_{n}(-\infty<n<\infty)$ & Homoclinic intersections \\
$\alpha$ & Set of all $\mathbf{P}_{n}$ 's and $\mathbf{Q}_{n}$ 's \\
$E_{n}, C_{n}(-\infty<n<\infty)$ & Escape and capture zones \\
$\mathcal{L}_{0}$ & Line of initial conditions \\
$\ell_{0}$ & Path-class of $\mathcal{L}_{0}$ \\
$D$ & Minimum delay time of the complex \\
$A, \partial A$ & Active region and boundary of the active region \\
$H_{n}(-\infty<n<\infty)$ & Holes in the active region \\
$A^{*}=A \backslash \cup_{n} H_{n}$ & Active region minus holes \\
$\mathcal{E}_{n}, \mathcal{U}_{n}$ & Paths along $\mathcal{S}$ and $\mathcal{U}$ boundaries of $E_{n}$ \\
$\mathcal{S}_{n}, \mathcal{C}_{n}$ & Paths along $\mathcal{S}$ and $\mathcal{U}$ boundaries of $C_{n}$ \\
$e_{n}, u_{n}, s_{n}, c_{n}$ & Path-classes of $\mathcal{E}_{n}, \mathcal{U}_{n}, \mathcal{S}_{n}, \mathcal{C}_{n}$ \\
$F$ & Path-class $c_{1} c_{2} \cdots c_{D}$ \\
$\Pi\left(A^{*}, \alpha\right)$ & Fundamental groupoid of path-classes in $A^{*}$ \\
& having base points in $\alpha$ \\
\hline
\end{tabular}

$=0.25, m=0.57$. Figure 1 shows a phase space portrait for this map, along with the line of initial conditions $\mathcal{L}_{0}$ considered here. The same map is plotted in Fig. 1, Paper I, but with a different line of initial conditions.

We review the basic picture of phase space transport described in Paper I and Refs. 18, 19, 21, and 22. The map $\mathcal{M}$ has an unstable fixed point ( $\mathrm{X}$ point) denoted $\mathbf{z}_{\mathrm{X}}$ and having Liapunov factor $\alpha>1$, which is the larger of the two eigenvalues of $\mathcal{M}$ linearized about $\mathbf{z}_{\mathrm{X}}$. The $\mathrm{X}$ point has an associated homoclinic tangle consisting of the branch $\mathcal{S}$ of the stable manifold and the branch $\mathcal{U}$ of the unstable manifold (Fig. 1). The complex is the region of phase space bounded on the north by the segment of $\mathcal{S}$ connecting the homoclinic intersection $\mathbf{P}_{0}$ to $\mathbf{z}_{X}$ and bounded on the south by the segment of $\mathcal{U}$ connecting $\mathbf{P}_{0}$ to $\mathbf{z}_{X}$. The forward and backward iterates $\mathbf{P}_{n}=\mathcal{M}^{n}\left(\mathbf{P}_{0}\right)$ are homoclinic intersections with the same sense as $\mathbf{P}_{0}$. The homoclinic intersection $\mathbf{Q}_{0}$ and its iterates $\mathbf{Q}_{n}=\mathcal{M}^{n}\left(\mathbf{Q}_{0}\right)$ have the opposite sense.

The escape zone $E_{0}$ is the lobe bounded by the segments of $\mathcal{S}$ and $\mathcal{U}$ joining $\mathbf{P}_{0}$ to $\mathbf{Q}_{0}$. It maps forward to the lobes $E_{n}, n \geqslant 0$, which all lie outside the complex, and backward to the lobes $E_{-n}, n>0$, which all intersect the complex. Similarly, the capture zone $C_{0}$ is the lobe bounded by the segments of $\mathcal{S}$ and $\mathcal{U}$ between $\mathbf{Q}_{-1}$ and $\mathbf{P}_{0}$. It maps forward to the lobes $C_{n}, n>0$, which all intersect the complex, and backward to the lobes $C_{-n}, n \geqslant 0$, which all lie outside the complex. Under one iterate of the map the escape zone $E_{-1}$ maps from inside to outside the complex and the capture zone $C_{0}$ maps from outside to inside the complex; the lobes $E_{-1}$ and $C_{0}$ together form what is called a turnstile. ${ }^{21,22}$ It is important to emphasize that all points which escape in $n$ iterates lie in the escape zone $E_{-n}$.

In the escape-time plot shown in Fig. 2, the number of iterates $n_{i}$ to escape is plotted as a function along the line of initial conditions $\mathcal{L}_{0}$. Figure 2 is analogous to Fig. 2 of Paper I, but for a different choice of $\mathcal{L}_{0}$. For a given $n_{i}$, the set of escaping points is partitioned into open intervals called escape segments; an escape segment is one connected component of $E_{-n_{i}} \cap \mathcal{L}_{0}$. For example, the first three escape seg- ments at $n_{i}=3,4,5$ in Fig. 2 correspond to the three intersections of $\mathcal{L}_{0}$ with the lobes $E_{-n}, n=3,4,5$, shown in Fig. 1.

The Epistrophe Theorem of Paper I says that the escapetime plot contains sequences of escape segments, called epistrophes. Several epistrophes are denoted by arrows in Fig. 2. The first epistrophe starts at $n_{i}=3$ and converges monotonically upward, containing one escape segment for each $n_{i}$ $\geqslant 3$. A second epistrophe begins at $n_{i}=9$ and converges downward upon the end point of the $n_{i}=3$ segment. We say that the $n_{i}=3$ segment "spawns" this epistrophe. Two more epistrophes are spawned at $n_{i}=10$ and converge upon the two end points of the $n_{i}=4$ segment. Similarly, the $n_{i}=5$ segment spawns two more epistrophes beginning at $n_{i}=11$.

The data in Fig. 2 suggest the following Epistrophe Start Rule: each end point of an escape segment at $n$ iterates spawns an epistrophe which begins at $n+\Delta$ iterates, where in this case $\Delta=6$. This recursive rule is formulated precisely by Theorem 1 in Sec. III F. In general, $\Delta=D+1$, where $D$ describes the global topology of the tangle (Sec. III A). The fact that $\Delta=6$ in Fig. 2 is a consequence of the fact that $E_{-3}$ intersects $C_{3}$ (and no earlier $C_{n}, n<3$ ) in Fig. 1 .

On the left of Fig. 2 are plotted the winding numbers $n_{w}$ of the escaping trajectories, i.e., the number of times a given trajectory winds around the central stable zone as it escapes to infinity. Notice that all segments of the epistrophe beginning at $n_{i}=3$ have winding number $n_{w}=1$. Similarly, all segments of the epistrophes spawned by the $n_{i}=3,4,5$ segments have winding number $n_{w}=2$. The data in Fig. 2 thus suggest that all segments of an epistrophe have the same winding number and that this number is one greater than the winding number of the segment which spawned the epistrophe. This rule will be precisely formulated and proved in a separate publication.

\section{HOMOTOPIC LOBE DYNAMICS}

We introduce a new kind of symbolic dynamics, where the symbol sequences refer to paths in the plane (rather than trajectories of the map). This symbolic dynamics allows us to identify and describe a minimal set of escape segments along an arbitrary line of initial conditions. The theory of homotopy is central to our development. ${ }^{23-25}$ Homotopy theory allows us to ignore the detailed positions of the stable and unstable manifolds and concentrate instead on their global topological structure. Homotopy theory also provides a natural algebraic framework for describing this global structure.

We consider a "saddle-center map" $\mathcal{M}$, which has a simple homoclinic tangle, as seen in Fig. 1 and described precisely by Assumptions $1-5$ in Paper I. ${ }^{26}$

\section{A. The homotopy groupoid}

We define the active region $A$ to be the union of the complex with all of its forward and backward iterates. By construction, it is an invariant region of the phase plane. The boundary of $A$, denoted $\partial A$, contains alternating segments of $\mathcal{S}$ and $\mathcal{U}$ (the outer boundaries of capture and escape zones) as well as the $\mathrm{X}$ point. ${ }^{27}$ The boundary $\partial A$ has a well-defined orientation determined by the orientations of $\mathcal{S}$ and $\mathcal{U}$. 


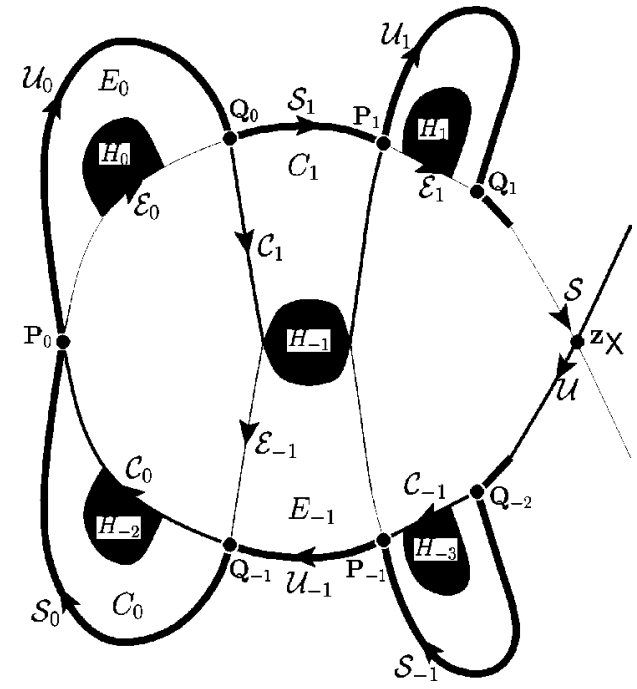

FIG. 3. Qualitative phase space portrait for the delay time $D=1$. Capture zone $C_{1}$ overlaps escape zone $E_{-1}$, so some orbits enter the complex on one iterate and leave on the next. The complex is bounded by the unstable manifold $\mathcal{U}$ from $\mathbf{z}_{\mathrm{X}}$ to $\mathbf{P}_{0}$ and by the stable manifold $\mathcal{S}$ from $\mathbf{P}_{0}$ back to $\mathbf{z}_{\mathrm{X}}$. The active region $A$ is the union of the complex with $E_{n}, n \geqslant 0$, and $C_{n}$, $n \leqslant 0$. The boundary $\partial A$ of $A$ contains alternating segments $\mathcal{S}_{n}$ and $\mathcal{U}_{n}$ of the stable and unstable manifolds. The inner boundaries of $E_{n}$ and $C_{n}$ are, respectively, denoted $\mathcal{E}_{n}$ and $\mathcal{C}_{n}$. The intersection of $C_{n}$ with $E_{n-2}$ is the "hole" $H_{n-2}$.

Let $D>0$ be the smallest integer such that $C_{D+1}$ intersects $E_{0}$. Considering all scattering trajectories which begin outside the complex, enter the complex, and eventually exit, $D$ is the smallest possible number of iterates spent inside the complex. For this reason, we call $D$ the minimum delay time of the complex or simply the delay time. The delay time is equivalently defined by the first pre-iterate $E_{-D+1}$ of $E_{0}$ which intersects $C_{0}$. In Fig. 1, the delay time $D$ is equal to 5 . (The delay time $D$ agrees with Easton's signature $\ell .^{17-19}$ )

For the case $D=1$, shown in Fig. 3, $E_{-1}$ intersects $C_{1}$, forming an open region $H_{-1}=E_{-1} \cap C_{1}$, which we view as a hole in the active region $A$. Mapping this hole backwards and forwards gives an infinite set of holes $H_{n}$. More generally, for arbitrary $D$ we define the holes $H_{n}=E_{n} \cap C_{n+\Delta}$, $-\infty<n<\infty$, where $\Delta=D+1$. (See Fig. 4 for the case $D$ =3.) The set $A^{*}=A \backslash \cup_{n} H_{n}$ is the active region minus all the holes $H_{n}$. The $D$ holes $H_{-D}, \ldots, H_{-1}$ are inside the complex; all other holes are outside.

The homoclinic intersections $\mathbf{P}_{n}$ and $\mathbf{Q}_{n},-\infty<n<\infty$, form a subset $\alpha$ of the boundary $\partial A$. Two paths (or directed curves) having the same initial and final points $\mathbf{s}_{0}, \mathbf{s}_{1} \in \alpha$ are said to be homotopic if one can be continuously distorted into the other without passing through a hole $H_{n}$ and without moving their end points. ${ }^{28}$ The concept of homotopy defines equivalence classes of paths; the path-class, or homotopy class, $a$ is the set of all paths homotopic to an arbitrary path $\mathcal{A} \in a$. That is, two paths belong to the same homotopy class if they can be distorted one into the other without changing the end points or passing through any hole; likewise, two homotopy classes $a$ and $b$ are equal if a path $\mathcal{A}$ in class $a$ can be distorted into a path $\mathcal{B}$ in class $b$.

We are particularly interested in the following paths. For each $n$, we define $\mathcal{S}_{n}$ to be the path along the $\mathcal{S}$ boundary of

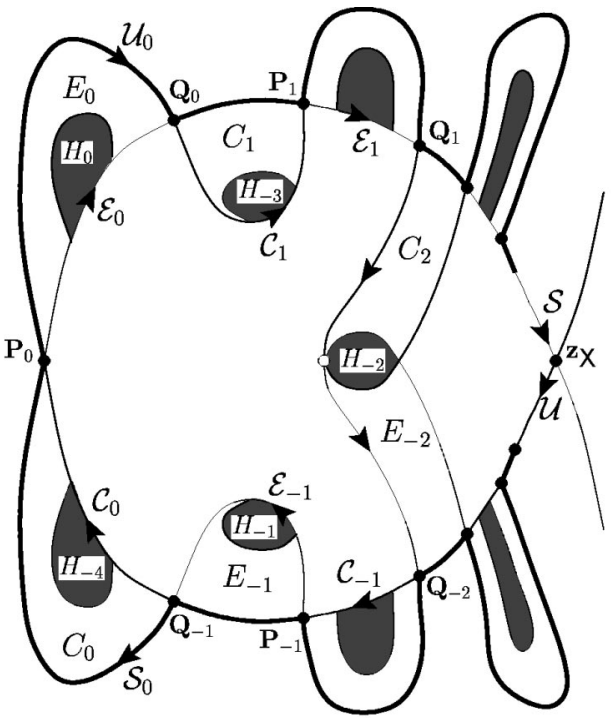

FIG. 4. Qualitative phase space portrait for delay time $D=3$. Here $C_{2}$ overlaps $E_{-2} \cdot \mathcal{C}_{n}$ and $\mathcal{S}_{n}$ link $\mathbf{Q}_{n-1}$ to $\mathbf{P}_{n}$ encircling hole $H_{n-4}$; likewise, $\mathcal{U}_{n}$ and $\mathcal{E}_{n}$ link $\mathbf{P}_{n}$ to $\mathbf{Q}_{n}$ encircling hole $H_{n}$.

capture zone $C_{n}$, joining $\mathbf{Q}_{n-1}$ to $\mathbf{P}_{n}$, and we define $\mathcal{U}_{n}$ to be the path along the $\mathcal{U}$ boundary of escape zone $E_{n}$, joining $\mathbf{P}_{n}$ to $\mathbf{Q}_{n}$, as shown in Fig. 3. These paths lie in the boundary $\partial A$ of the active region. Similarly, for each $n$, we define $\mathcal{E}_{n}$ to be the path along the $\mathcal{S}$ boundary of $E_{n}$ and $\mathcal{C}_{n}$ to be the path along the $\mathcal{U}$ boundary of $C_{n}$. These paths bound the lobes $E_{n}$ and $C_{n}$ in the interior of $A$. Since each path $\mathcal{E}_{n}, \mathcal{C}_{n}, \mathcal{U}_{n}$, and $\mathcal{S}_{n}$ has end points in $\alpha$ and does not pass through any of the holes $H_{n}$, each belongs to a well-defined homotopy class. These classes are distinct, since none of the curves can be distorted into any other, and we denote them by $e_{n}, c_{n}, u_{n}$, and $s_{n}$, respectively. These homotopy classes encode global topological information about the structure of the tangle.

Let $\Pi\left(A^{*}, \alpha\right)$ be the collection of all homotopy classes of paths in $A^{*}$ having end points in $\alpha$. For a path-class $a_{1}$ $\in \Pi\left(A^{*}, \alpha\right)$ joining $\mathbf{s}_{0}$ to $\mathbf{s}_{1}$ and a path-class $a_{2} \in \Pi\left(A^{*}, \alpha\right)$ joining $\mathbf{s}_{1}$ to $\mathbf{s}_{2}$, their product $a_{1} a_{2}$ joins $\mathbf{s}_{0}$ to $\mathbf{s}_{2}$ and is constructed by first traversing a representative path $\mathcal{A}_{1} \in a_{1}$ followed by a representative $\mathcal{A}_{2} \in a_{2}$. The homotopy class of a constant path, i.e., one which remains at a given point $\mathbf{s}$ $\in \alpha$ for all times, is denoted 1 (with the end point $\mathbf{s} \in \alpha$ being understood from context); for all $a \in \Pi\left(A^{*}, \alpha\right), 1 a=a 1$ $=a$. For a class $a \in \Pi\left(A^{*}, \alpha\right)$, its inverse $a^{-1}$ contains a representative path from $a$, but traversed backwards; clearly, $a a^{-1}=1$. The set $\Pi\left(A^{*}, \alpha\right)$ thus has most of the structure of a group (multiplication, identity, and inverse) except in one respect: the product $a_{1} a_{2}$ is not defined between arbitrary elements $a_{1}$ and $a_{2}$ but only between elements such that the final point of $a_{1}$ equals the initial point of $a_{2}$. A set with such a restricted product is called a groupoid, ${ }^{29}$ and $\Pi\left(A^{*}, \alpha\right)$ is called the fundamental groupoid of path-classes in $A^{*}$ having base points in $\alpha$.

The dynamical map $\mathcal{M}$, acting on points in the plane, induces a map on the path-classes, which forms a kind of symbolic dynamics on the symbols $e_{n}, c_{n}, u_{n}$, and $s_{n}$. 
When $\mathcal{M}$ acts on these elements, it simply shifts their indices,

$$
\begin{aligned}
& \mathcal{M}\left(e_{n}\right)=e_{n+1}, \\
& \mathcal{M}\left(c_{n}\right)=c_{n+1}, \\
& \mathcal{M}\left(u_{n}\right)=u_{n+1}, \\
& \mathcal{M}\left(s_{n}\right)=s_{n+1} .
\end{aligned}
$$

\section{B. The minimal set of escape segments}

We now turn our attention to the line of initial conditions, which we assume is given by a path $\mathcal{L}_{0}$ that (1) has end points $\lambda_{i}, \lambda_{f} \in \partial A$, (2) does not self-intersect, and (3) does not intersect any hole $H_{n}$ or the $\mathrm{X}$ point $\mathbf{z}_{\mathbf{X}} \cdot{ }^{30}$ For the homotopy analysis, we must shift the end points of $\mathcal{L}_{0}$ so that they lie in the set $\alpha$. For example, we shift the initial point by first traversing a path $\mathcal{K}_{i}$ before traversing $\mathcal{L}_{0} ; \mathcal{K}_{i}$ begins at one of the two points in $\alpha$ on either side of $\lambda_{i}$, runs along $\partial A$, and finally terminates at $\lambda_{i}$. By thus shifting both end points, we assign to $\mathcal{L}_{0}$ a well-defined homotopy class $\ell_{0} \in \Pi\left(A^{*}, \alpha\right){ }^{31}$

The intersection of $\mathcal{L}_{0}$ with escape zone $E_{-n}$ is the set of points that escape on the $n$th iterate, and any connected component of this set is called an escape segment; sometimes we will use the term $e_{-n}$-segment to emphasize an intersection with $E_{-n}$. (The index $n$ may, in fact, be either positive or negative.) In this article, we answer the following two questions regarding a minimal set of escape segments.

Question 1: What is the minimum number of intersections possible between a representative path $\mathcal{L}_{0}^{\prime} \in \ell_{0}$ and a representative path $\mathcal{E}_{-n}^{\prime} \in e_{-n}$ ?

The minimum number of $e_{-n}$-segments is half the minimum number of intersections.

Question 2: Let $\mathcal{L}_{0}^{\prime} \in \ell_{0}, \mathcal{E}_{-n_{1}}^{\prime} \in e_{-n_{1}}, \mathcal{E}_{-n_{2}}^{\prime} \in e_{-n_{2}}\left(n_{1}\right.$ $\left.\neq n_{2}\right)$ be paths which minimize all possible pairwise- and self-intersections. In particular, $\mathcal{E}_{-n_{1}}^{\prime}$ and $\mathcal{E}_{-n_{2}}^{\prime}$ do not intersect each other or themselves, and $\mathcal{L}_{0}^{\prime}$ has the minimum number of escape segments at both $n_{1}$ and $n_{2}$ iterates. What are the positions of the escape segments at $n_{1}$ iterates relative to those at $n_{2}$ iterates? $?^{32,34}$

In answering these questions we allow ourselves to distort $\mathcal{L}_{0}$ and $\mathcal{E}_{-n}$ into paths $\mathcal{L}_{0}^{\prime}$ and $\mathcal{E}^{\prime}{ }_{-n}$ to minimize the number of intersections. Thus we are constructing a "distorted escape zone" $E_{-n}^{\prime}$ whose intersection with $\mathcal{L}_{0}^{\prime}$ is a set of "distorted escape segments." Henceforth, we omit the descriptor "distorted" and leave it understood.

The answer to the above two questions will be obtained from the algebraic algorithm in Sec. III D, which will lead in turn to a proof of the Epistrophe Start Rule in Sec. III F.

\section{The untangled basis of path-classes}

By a basis of a groupoid we mean a minimal set of elements that generate the entire groupoid. To construct a basis of the fundamental groupoid $\Pi\left(A^{*}, \alpha\right)$, we first include the path-classes $\left(\ldots, s_{-1}, s_{0}, s_{1}, \ldots ; \ldots, u_{-1}\right.$, $\left.u_{0}, u_{1}, \ldots\right)$ along the boundary of the active region $\partial A$.

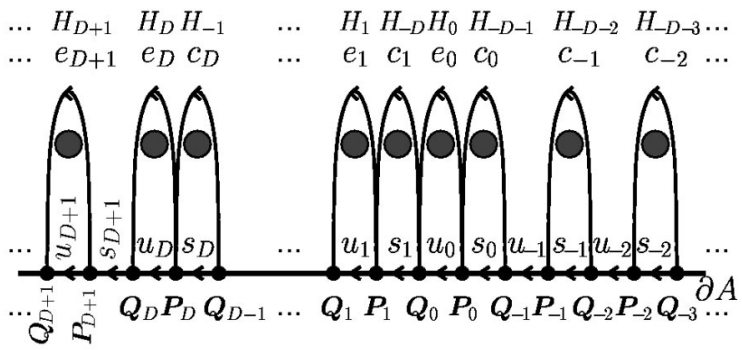

FIG. 5. Basis paths are shown for the active region $A^{*}$ with an infinite number of holes $H_{n}$ punctured in it. $A^{*}$ is bounded below by $\partial A$, which has been straightened into a line. The basis of $\Pi\left(A^{*}, \alpha\right)$ contains the pathclasses $s_{n}$ and $u_{n}(-\infty<n<\infty)$ which link $\mathbf{Q}_{n-1}$ to $\mathbf{P}_{n}$ and $\mathbf{P}_{n}$ to $\mathbf{Q}_{n}$ along $\partial A$. The basis also includes the path-classes $c_{n},-\infty<n \leqslant D$ (bounding capture zones) and $e_{n}, 0 \leqslant n<\infty$ (bounding escape zones). The classes $e_{n}$, $0 \leqslant n$, encircle the holes $H_{n}$, and the classes $c_{n}, n \leqslant D$, encircle the holes $H_{-D+n-1}$. (Note that the ordering of the holes shown in the diagram does not coincide with the order of their indices.) For $D=1$ or 3 , the reader may verify that the curves drawn above agree topologically with those in Figs. 3 and 4 .

We then select path-classes $\left(\ldots, c_{-1}, c_{0} ; c_{1}, \ldots\right.$, $\left.c_{D} ; e_{0}, e_{1}, \ldots\right)$ that encircle the holes in $A^{*}$, so that the complete basis is

$$
\begin{aligned}
& \left(\ldots, c_{-1}, c_{0} ; c_{1}, \ldots, c_{D} ; e_{0}, e_{1}, \ldots ;\right. \\
& \left.\ldots, s_{-1}, s_{0}, s_{1}, \ldots ; \ldots, u_{-1}, u_{0}, u_{1}, \ldots\right),
\end{aligned}
$$

shown schematically in Fig. 5. The elements $c_{1}, \ldots, c_{D}$ are special in that they are the only basis elements which must enter the interior of the complex, encircling the $D$ holes $H_{-D}, \ldots, H_{-1}$.

The representative paths $\mathcal{C}_{n}, \mathcal{E}_{n}, \mathcal{S}_{n}$, and $\mathcal{U}_{n}$ for this basis satisfy (see Fig. 5): (1) no path in the basis intersects itself or any other path in the basis (except perhaps at the end points); (2) each representative $\mathcal{E}_{n}$ and $\mathcal{C}_{n}$ in the basis encircles exactly one hole, and each hole is encircled exactly once. Furthermore, (3) all homotopy classes of relevance to us, specifically $\ell_{0}, c_{n}$, and $e_{n},-\infty<n<\infty$, have a unique finite reduced expansion in the basis. ${ }^{33}$ (A reduced expansion is a sequence of elements in which any two adjacent factors $a$ and $a^{-1}$ have been canceled.) Because of these properties and the simple picture shown in Fig. 5, we call this basis the "untangled basis."

\section{Symbolic dynamics of path-classes}

Now we develop the symbolic dynamics that will describe the minimal set of escape segments. First, however, we must assign a direction to each escape segment. Recall that the two end points of an $e_{n}$-segment $(-\infty<n<\infty)$ are intersection points between a path $\mathcal{E}_{n}^{\prime} \in e_{n}$ and a path $\mathcal{L}^{\prime}$ $\in \ell$. Using the orientation of $\mathcal{E}_{n}^{\prime}$, one of these end points occurs first. We define the direction of an escape segment to point along $\mathcal{L}^{\prime}$ from the second end point to the first end point. (See Fig. 6.) Recall that $\mathcal{L}^{\prime}$ has an independent direction defined by its own parametrization. An escape segment is said to "point forward" if its direction is the same as $\mathcal{L}^{\prime}$ and to "point backward" otherwise. A point on $\mathcal{L}^{\prime}$ is said to 


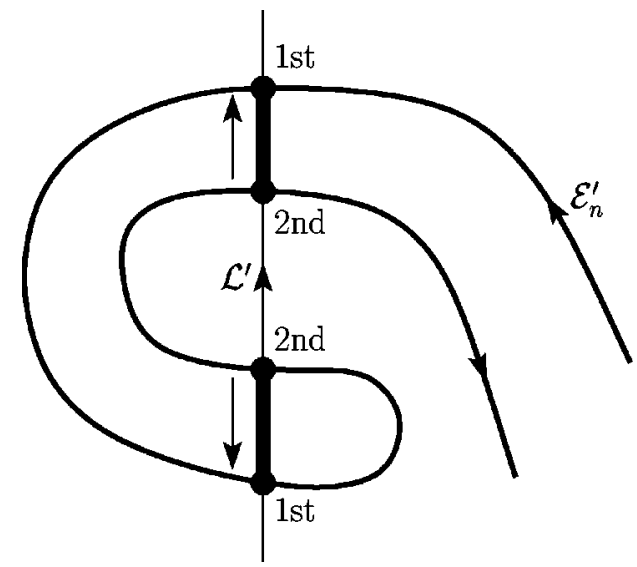

FIG. 6. We illustrate the convention for assigning a direction to each escape segment. Each of the two bold segments shown has a first and second end point; the first end point precedes the second end point as one moves forward along the path $\mathcal{E}_{n}^{\prime}$. The direction of each segment points from the second to the first end point.

lie on the "positive" side of an escape segment if the segment points toward it and on the "negative" side otherwise.

We need the forward iterates of all untangled basis elements expressed in terms of the untangled basis. For most elements, this is given by Eq. (1). Only one additional equation is needed,

$$
\mathcal{M}\left(c_{D}\right)=c_{D+1}=F^{-1} u_{0}^{-1} e_{0} F s_{D+1},
$$

where $F$ is an abbreviation for the path-class

$$
F=c_{1} e_{1} c_{2} e_{2} \ldots c_{D} e_{D} .
$$

Notice that the right-hand side of Eq. (3) [after substituting in Eq. (4)] is expressed entirely in terms of the untangled basis (2). Equation (3) is proved by first observing

$$
e_{-1}=u_{-1}\left(c_{0} e_{0} F e_{D}^{-1}\right) s_{D}^{-1} c_{D}\left(e_{D} F^{-1} e_{0}^{-1} c_{0}^{-1}\right),
$$

which, though rather lengthy, can be directly verified from a figure such as 3 or 4 ; one simply concatenates the basis paths as shown on the right and then distorts the resulting path into $\mathcal{E}_{-1} \in e_{-1}$. By applying $\mathcal{M}$ to both sides of Eq. (5) and solving for $c_{D+1}$, one obtains Eq. (3). It is convenient to explicitly compute the forward iterate of $F$ from Eq. (3),

$$
\mathcal{M}(F)=e_{1}^{-1} c_{1}^{-1} u_{0}^{-1} e_{0} F s_{D+1} e_{D+1} .
$$

For the purpose of computing the minimal set of escape segments, the $e_{n}$ basis elements $(n \geqslant 0)$ and the $s_{n}$ basis elements (all $n$ ) can simply be omitted from any expression that contains them, resulting in significant computational simplification; for example, Eqs. (3), (4), and (6) above become Eqs. (7)-(9) below. This is explained more fully in Appendix A. We can now state the algorithm for constructing the minimal set of escape segments up to a given iterate $N$.

Algorithm 1: Let $\mathcal{L}_{0}$ be the line of initial conditions and $\ell_{0} \in \Pi\left(A^{*}, \alpha\right)$ its homotopy class.

(1) Expand $\ell_{0}$ in the untangled basis, omitting any $e_{n}$-factors for $n \geqslant 0$ and all $s_{n}$-factors for $-\infty<n<\infty$.

(2) Compute $\ell_{N}$ by iterating $\ell_{0}$ forward $N$ times using Eqs. (1b) and (1c), and
$\mathcal{M}\left(c_{D}\right)=F^{-1} u_{0}^{-1} F$,

where

$F=c_{1} c_{2} \cdots c_{D}$.

For convenience, one may also use the following formula, which explicitly maps $F$ forward:

$\mathcal{M}(F)=c_{1}^{-1} u_{0}^{-1} F$.

Carry out any cancellations of factors using $a a^{-1}=1$, so that $\ell_{N}$ is expressed as a reduced expansion in the untangled basis.

Then,

(a) Each $u_{n}$ or $u_{n}^{-1}$ factor $(n \geqslant 0)$ in the expansion of $\ell_{N}$ corresponds to a segment that escapes in $n_{i}=N-n$ iterates and that points backwards or forwards, respectively.

(b) The relative positions of the $u_{n}$-factors in the expansion of $\ell_{N}$ are the same as the relative positions of their corresponding escape segments along $\mathcal{L}_{0}$.

This algorithm is justified in Appendix A.

\section{E. Examples}

We apply Algorithm 1 to compute the minimal set of escape segments (up to $n_{i}=3$ ) for the simple example $D$ $=1, \ell_{0}=c_{D}=c_{1}$. Carrying out step 2, the first three iterates of $\ell_{0}$ are computed to be

$$
\begin{aligned}
& \ell_{0}=c_{1}, \\
& \ell_{1}=c_{1}^{-1} \underline{u_{0}^{-1} c_{1},} \\
& \ell_{2}=c_{1}^{-1} \underline{u_{0} c_{1}} \underline{u_{1}^{-1} c_{1}^{-1} \underline{u_{0}^{-1} c_{1},}} \\
& \ell_{3}=c_{1}^{-1} \underline{u_{0}} c_{1} \underline{u_{1}} c_{1}^{-1} \underline{u_{0}^{-1} c_{1}} \underline{u_{2}^{-1}} c_{1}^{-1} \underline{u_{0} c_{1} u_{1}^{-1} c_{1}^{-1} u_{0}^{-1} c_{1},}
\end{aligned}
$$

where the $u_{n}$-factors have been underlined for greater visibility. We now consider the consequences of results $\mathrm{A}$ and $\mathrm{B}$ in the algorithm. Examining $\ell_{1}$, it contains a single factor $u_{0}^{-1}$, which yields a single forward pointing escape segment at $n_{i}=1$, as shown in Fig. 7(a). Iterating forward to $\ell_{2}$, this $n_{i}=1$ escape segment corresponds to the factor $u_{1}^{-1}$ in Eq. (10c); on either side of this factor are factors $u_{0}$ and $u_{0}^{-1}$, corresponding, respectively, to backward and forward pointing segments that escape at $n_{i}=2$. Iterating once more, $\ell_{3}$ has four $u_{0}$-factors (either $u_{0}$ or $u_{0}^{-1}$ ) corresponding to four escape segments at $n_{i}=3$ and with relative positions and directions shown in Fig. 7(a).

Considering now an arbitrary $D, \ell_{0}=c_{D}$ propagates forward as

$$
\begin{aligned}
& \ell_{0}=c_{D}, \\
& \ell_{1}=c_{D+1}=F^{-1} \underline{u_{0}^{-1} F,} \\
& \ell_{2}=c_{D+2}=\left(F^{-1} \underline{u_{0}} c_{1}\right) \underline{u_{1}^{-1}}\left(c_{1}^{-1} u_{0}^{-1} F\right), \\
& \ell_{3}=c_{D+3}=\left(F^{-1} \underline{u_{0}} c_{1} \underline{u_{1}} c_{2}\right) \underline{u_{2}^{-1}}\left(c_{2}^{-1} u_{1}^{-1} c_{1}^{-1} u_{0}^{-1} F\right),
\end{aligned}
$$




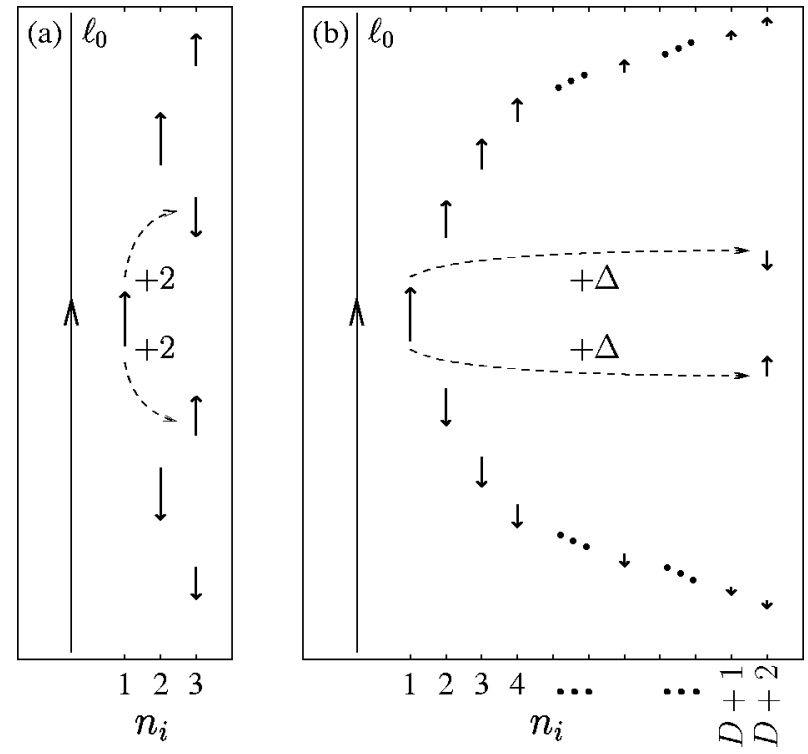

FIG. 7. The escape-time plots are shown qualitatively for $\ell_{0}=c_{D}$ where (a) $D=1$ and (b) $D$ is arbitrary. At each $n_{i}$, a segment (in the minimal set) that escapes in $n_{i}$ iterates is represented by an arrow giving the direction of the segment; $\ell_{0}$ itself points up. The minimal set is determined by Eq. (10d) for (a) and Eq. (11h) for (b). Note that two new segments are spawned $\Delta=D$ +1 iterates beyond the first segment, an example of the Epistrophe Start Rule.

$$
\begin{aligned}
\ell_{4}= & c_{D+4}=\left(F^{-1} u_{0} c_{1} u_{1} c_{2} u_{2} c_{3}\right) \underline{u_{3}^{-1}} \\
& \times\left(c_{3}^{-1} \underline{\left.u_{2}^{-1} c_{2}^{-1} u_{1}^{-1} c_{1}^{-1} u_{0}^{-1} F\right),}\right. \\
\vdots & \\
\ell_{n}= & c_{D+n}=\left(F^{-1} u_{0} c_{1} u_{1} c_{2} \ldots \underline{u_{n-2}} c_{n-1}\right) \underline{u_{n-1}^{-1}} \\
& \times\left(c_{n-1}^{-1} \underline{u_{n-2}^{-1}} \ldots c_{2}^{-1} u_{1}^{-1} c_{1}^{-1} u_{0}^{-1} F\right),
\end{aligned}
$$

$\vdots$

$$
\begin{aligned}
& \ell_{D+1}=c_{2 D+1}=\left(F^{-1} u_{0} c_{1} \underline{u_{1}} c_{2} \ldots \underline{u_{D-1}} c_{D}\right) u_{D}^{-1} \\
& \times\left(c_{D}^{-1} u_{D-1}^{-1} \ldots c_{2}^{-1} u_{1}^{-1} c_{1}^{-1} u_{0}^{-1} F\right), \\
& \ell_{D+2}=c_{2 D+2}=\left(F^{-1} u_{0} c_{1} u_{1} c_{2} \ldots u_{D-1} c_{D}\right) \\
& \times\left(u_{D} F^{-1} u_{0}^{-1} F u_{D+1}^{-1} F^{-1} u_{0} F u_{D}^{-1}\right) \\
& \times\left(c_{D}^{-1} u_{D-1}^{-1} \ldots c_{2}^{-1} u_{1}^{-1} c_{1}^{-1} u_{0}^{-1} F\right) .
\end{aligned}
$$

The minimal set of escape segments for $\ell_{0}=c_{D}$, as constructed from results $A$ and $B$ in the algorithm, is shown schematically in Fig. 7(b). The set contains an upward- and a downward-converging epistrophe, with two additional segments at $n_{i}=D+2$. These two segments are the beginnings of two new epistrophes spawned $\Delta=D+1$ iterates after the first segment. This spawning behavior is also visible in Fig. 7(a) for $\Delta=D+1=2$. In the next section we show that all lines of initial conditions have a minimal set that eventually displays such spawning behavior.

\section{F. The Epistrophe Start Rule}

After a certain number of iterates, the minimal set for any $\mathcal{L}_{0}$ has a simple recursive structure described by the following theorem, which is proved in Appendix B.

Theorem 1: Let $\mathcal{M}$ be a "saddle-center map" satisfying Assumptions 1-5 of Paper I (Ref. 26) and having an arbitrary minimum delay time $D \geqslant 1$. Let $\mathcal{L}_{0}$ be the line of initial conditions. There exists some iterate $N_{0}>0$ such that the minimal set of escape segments at all $N \geqslant N_{0}$ iterates can be constructed from the following two recursive rules.

Epistrophe Continuation Rule: Every segment (in the minimal set) that escapes at $N-1$ iterates has on its immediate positive side a segment that escapes at $N$ iterates and which has the same direction.

Epistrophe Start Rule: Every segment that escapes at $N-\Delta$ iterates $(\Delta=D+1)$ spawns immediately on both of its sides a segment that escapes at $N$ iterates and which points toward the spawning segment.

Explicitly, $N_{0}=\max \left\{-n_{c}+1,-n_{u}, 0\right\}+D+2$, where $n_{c}$ and $n_{u}$ are, respectively, the lowest indices of the $c_{n^{-}}$and $u_{n}$-factors in the expansion of the path-class $\ell_{0}$ of $\mathcal{L}_{0}$ in the untangled basis.

To say that an $e_{n_{1}}$-segment lies "on the immediate positive/negative side of" an $e_{n_{2}}$-segment means that in the minimal set there is no earlier $e_{n_{3}}$-segment, $n_{3}$ $\leqslant \max \left\{n_{1}, n_{2}\right\}$, between the two. Notice that new epistrophes are spawned by the Epistrophe Start Rule; the Epistrophe Continuation Rule simply propagates those epistrophes started earlier. Notice also that segments of an epistrophe point in the direction of convergence of the epistrophe. The early structure of the minimal set (before $N_{0}$ ) can be computed using the algorithm in Sec. III D. Thus, the algorithm gives the early behavior of the minimal set, and the simpler recursive rules give the subsequent behavior.

\section{EXAMPLES}

Using the map $\mathcal{M}$ discussed in Sec. II and illustrated by Fig. 1, we consider the escape-time plots for two different lines of initial conditions.

\section{A. Line 1}

We consider the line of initial conditions $\mathcal{L}_{0}$ in Fig. 1. First we determine the homotopy class of $\mathcal{L}_{0}$. Since neither end point of $\mathcal{L}_{0}$ is in $\alpha$, we must shift each end point as described in Sec. III B. Since the initial (southernmost) end point is on the curve $\mathcal{U}_{-3}$ (the southern boundary of $E_{-3}$ ), we can shift it either east to $\mathbf{P}_{-3}$ or west to $\mathbf{Q}_{-3}$; we choose $\mathbf{Q}_{-3}$ since this will guarantee that the beginning of $\mathcal{L}_{0}$ still intersects $E_{-3}$. Since the final (northernmost) end point is on the curve $\mathcal{S}_{3}$ (the northern boundary of $C_{3}$ ), it does not matter whether we shift it east to $\mathbf{P}_{3}$ or west to $\mathbf{Q}_{2}$; we choose $\mathbf{Q}_{2}$. Following step 1 in the algorithm, we scrutinize Fig. 1 to determine that the homotopy class $\ell_{0} \in \Pi\left(A^{*}, \alpha\right)$ of the adjusted curve is $\ell_{0}=c_{-2} u_{-2} c_{-1} u_{-1} c_{0} e_{0} F e_{5}^{-1} s_{5}^{-1} e_{4}^{-1} s_{4}^{-1}$ $\times e_{3}^{-1} s_{3}^{-1}$. (The reader is invited to verify this by drawing a 
large copy of Fig. 1, putting in the holes, and constructing the curve resulting from the formula.) After omitting $e_{n}$ - and $s_{n}$-factors, this simplifies to

$$
\ell_{0}=c_{-2} u_{-2} c_{-1} u_{-1} c_{0} F .
$$

Following step 2, we map $\ell_{0}$ forward using Eqs. (1b), (1c), (7), and (9) with $D=5$,

$$
\begin{aligned}
\ell_{1}= & c_{-1} u_{-1} c_{0} F, \\
\ell_{2}= & c_{0} F, \\
\ell_{3}= & \underline{u_{0}^{-1} F} \\
\ell_{4}= & u_{1}^{-1} c_{1}^{-1} u_{0}^{-1} F, \\
\ell_{5}= & u_{2}^{-1} c_{2}^{-1} u_{1}^{-1} c_{1}^{-1} \underline{u_{0}^{-1} F}, \\
\ell_{6}= & u_{3}^{-1} c_{3}^{-1} u_{2}^{-1} c_{2}^{-1} u_{1}^{-1} c_{1}^{-1} u_{0}^{-1} F, \\
\ell_{7}= & u_{4}^{-1} c_{4}^{-1} u_{3}^{-1} c_{3}^{-1} u_{2}^{-1} c_{2}^{-1} u_{1}^{-1} c_{1}^{-1} u_{0}^{-1} F, \\
\ell_{8}= & u_{5}^{-1} c_{5}^{-1} u_{4}^{-1} c_{4}^{-1} u_{3}^{-1} c_{3}^{-1} u_{2}^{-1} c_{2}^{-1} u_{1}^{-1} c_{1}^{-1} u_{0}^{-1} F, \\
\ell_{9}= & u_{6}^{-1} F^{-1} u_{0} F u_{5}^{-1} c_{5}^{-1} u_{4}^{-1} c_{4}^{-1} u_{3}^{-1} c_{3}^{-1} \\
& \times u_{2}^{-1} c_{2}^{-1} u_{1}^{-1} c_{1}^{-1} u_{0}^{-1} F .
\end{aligned}
$$

For greater visibility, we have underlined each $u_{0}$-factor. Mapping forward once more, we find

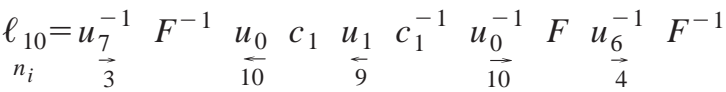

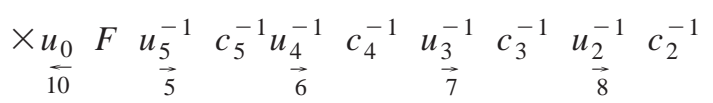

$$
\begin{aligned}
& \times \underset{\overrightarrow{9}}{u_{1}^{-1}} c_{1}^{-1} \quad \underset{10}{u_{0}^{-1}} F .
\end{aligned}
$$

Below each $u_{n}$-factor in Eq. (14), we have recorded the number of iterates to escape; the arrow indicates whether the segment is forward- or backward-pointing. The results of Eqs. (13) and (14) are shown qualitatively in Fig. 8(a); they should be compared with the calculation in Fig. 2. We examine these results in detail.

(1) As stated in Algorithm 1, each $u_{n}$ or $u_{n}^{-1}$ factor in $\ell_{N}$ corresponds to a segment of $\mathcal{L}_{0}$ that escapes in $n_{i}=N-n$ iterates. Equation (14) gives the minimal set of escape segments up to $n_{i}=10$. (2) After a certain iterate $N_{0}$, we can determine the minimal set using the Epistrophe Continuation Rule and Epistrophe Start Rule in Theorem 1. Explicitly, $N_{0}=\max \left\{-n_{c}+1,-n_{u}, 0\right\}+D+2$; examining Eq. (12) we see $n_{c}=n_{u}=-2$, and since $D=5, N_{0}=10$. So, for all iterates $n_{i} \geqslant 10$, Algorithm 1 and Theorem 1 give identical results. (3) Direct computation (Fig. 2) indicates that up to $n_{i}$ $=14$, there are no additional escape segments outside the minimal set. The first segment in the computation which is not in the minimal set is indicated by an asterisk in Fig. 2 at $n_{i}=15$; it is an example of what we call a strophe. (4) No epistrophe converges upon the lower end point of the $n_{i}=3$
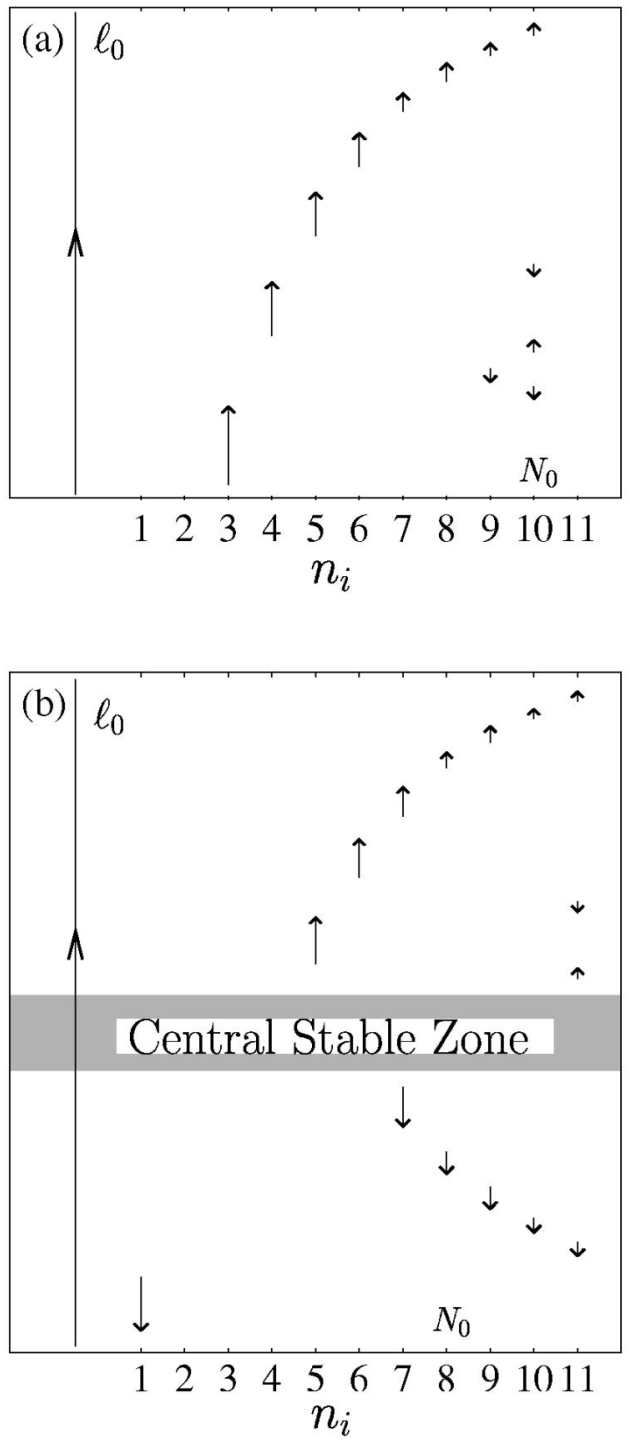

FIG. 8. The escape-time plots are shown qualitatively for two example lines of initial conditions: (a) and (b) are determined by Eqs. (14) and (17), respectively. In each plot we indicate the value of $N_{0}$ after which all segments in the minimal set can be deduced using Theorem 1 .

segment, either in the minimal set [Fig. 8(a)] or the numerical data (Fig. 2), because this point is an intersection between $\mathcal{L}_{0}$ and the unstable manifold.

\section{B. Line 2}

We consider the line of initial conditions $\mathcal{L}_{0}$ in Fig. 1 of Paper I. In order to define the homotopy class of this line, it must first be adjusted. From Fig. 1, Paper I, we see that $\mathcal{L}_{0}$ intersects the holes $H_{-1}=E_{-1} \cap C_{5}$ and $H_{-5}=E_{-5} \cap C_{1}$. We adjust $\mathcal{L}_{0}$ within each of these holes so that it runs along the boundary of the hole, on either the east or west side, and not through the hole itself. For the northern hole $H_{-5}$, we adjust $\mathcal{L}_{0}$ to run along the eastern boundary, so that it still passes through $E_{-5}$. For the southern hole $H_{-1}$, we adjust $\mathcal{L}_{0}$ to run along the western boundary. As in Sec. IV A, the 
end points of $\mathcal{L}_{0}$ must also be adjusted, so that they lie in $\alpha$. We shift the southern end point to $\mathbf{P}_{-1}$ and the northern end point to $\mathbf{P}_{1}$.

The homotopy class $\ell_{0} \in \Pi\left(A^{*}, \alpha\right)$ of the adjusted curve is $\ell_{0}=u_{-1} c_{0} e_{0} c_{1}$, which simplifies to

$$
\ell_{0}=u_{-1} c_{0} c_{1} \text {. }
$$

Then $\ell_{0}$ maps forward as

$$
\begin{aligned}
\ell_{1}= & u_{0} c_{1} c_{2} \\
\ell_{2}= & u_{1} c_{2} c_{3} \\
\ell_{3}= & u_{2} c_{3} c_{4} \\
\ell_{4}= & u_{3} c_{4} c_{5} \\
\ell_{5}= & u_{4} c_{5} F^{-1} u_{0}^{-1} F \\
\ell_{6}= & u_{5} F^{-1} c_{1} u_{1}^{-1} c_{1}^{-1} u_{0}^{-1} F \\
\ell_{7}= & u_{6} F^{-1} u_{0} c_{1} c_{2} u_{2}^{-1} c_{2}^{-1} u_{1}^{-1} c_{1}^{-1} u_{0}^{-1} F \\
\ell_{8}= & u_{7} F^{-1} u_{0} c_{1} u_{1} c_{2} c_{3} u_{3}^{-1} c_{3}^{-1} u_{2}^{-1} c_{2}^{-1} u_{1}^{-1} c_{1}^{-1} u_{0}^{-1} F \\
\ell_{9}= & u_{8} F^{-1} u_{0} c_{1} u_{1} c_{2} u_{2} c_{3} c_{4} \\
& \times u_{4}^{-1} c_{4}^{-1} u_{3}^{-1} c_{3}^{-1} u_{2}^{-1} c_{2}^{-1} u_{1}^{-1} c_{1}^{-1} u_{0}^{-1} F \\
\ell_{10}= & u_{9} F^{-1} \frac{u_{0}}{c_{1}} u_{1} c_{2} u_{2} c_{3} u_{3} c_{4} c_{5} \\
& \times u_{5}^{-1} c_{5}^{-1} u_{4}^{-1} c_{4}^{-1} u_{3}^{-1} c_{3}^{-1} u_{2}^{-1} c_{2}^{-1} u_{1}^{-1} c_{1}^{-1} u_{0}^{-1} F,
\end{aligned}
$$

and

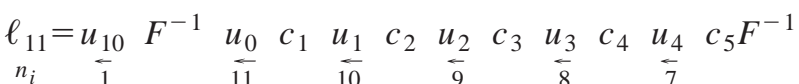

$$
\begin{aligned}
& \times \underset{\overrightarrow{11}}{u_{0}^{-1}} F \underset{\overrightarrow{5}}{u_{6}^{-1} F^{-1}} \quad \underset{11}{u_{0}} F \underset{\overrightarrow{6}}{u_{5}^{-1}} c_{5}^{-1} u_{\frac{\overrightarrow{7}}{7}}^{u_{4}^{-1}} c_{4}^{-1} \\
& \times \underset{\overrightarrow{8}}{u_{3}^{-1}} c_{3}^{-1} u_{\overrightarrow{9}}^{u_{2}^{-1}} c_{2}^{-1} \underset{\overrightarrow{10}}{u_{1}^{-1}} c_{1}^{-1} \underset{\overrightarrow{11}}{u_{0}^{-1}} F \text {. }
\end{aligned}
$$

The data from Eq. (17) are summarized in Fig. 8(b). This should be compared with the numerical calculation in Fig. 2 of Paper I.

Equation (17) gives the minimal set of escape segments up to $n_{i}=11$. In this case, examining Eq. (15), $n_{c}=0$ and $n_{u}=-1$, yielding $N_{0}=\max \{1,1,0\}+5+2=8$. Therefore for $n_{i} \geqslant 8$, the minimal set can be generated from Theorem 1 rather than the algorithm. The first numerically computed segment which is not in the minimal set (a strophe) does not occur until $n_{i}=16$; it is indicated by an asterisk in Fig. 2 of Paper I. As above, no epistrophe converges upon the lower end point of the $n_{i}=1$ segment because it is an intersection between $\mathcal{L}_{0}$ and the unstable manifold.

\section{CONCLUSIONS}

The results of the present paper combine with the results of Paper $\mathrm{I}^{1}$ to create a detailed picture of escape-time plots. On the one hand, the present study predicts the existence of a minimal set of escape segments (Algorithm 1). After some number of iterates, this set has a simple recursive pattern (Theorem 1) described by: (1) at each iterate, add new segments that perpetuate all earlier epistrophes; (2) at $\Delta=D$ +1 iterates after a given segment, spawn two new epistrophes on either side of this segment. These results say nothing about the lengths of segments or the separation between segments, and in particular say nothing about convergence properties of epistrophes. On the other hand, the results of Paper I do address such issues, and we find that epistrophes converge geometrically upon the end points of the segments that spawn them and furthermore that all epistrophes differ asymptotically by an overall scale factor (Epistrophe Theorem, Paper I).

The minimal set of escape segments typically omits some segments (strophes) that appear in the actual numerically computed escape-time plot. Nevertheless, the results of Paper I apply to such strophic segments as well. There will be an epistrophe which converges upon an end point of a strophe (Epistrophe Theorem). However, we cannot in general predict at which iterate such an epistrophe will begin. On the other hand, the numerical evidence of Fig. 2 and of Fig. 2 in Paper I is suggestive that even in this case, the epistrophes often begin $\Delta$ iterates beyond the strophe.

Strophes occur due to structure in the lobes $E_{-n}$ that we have ignored in our simple topological picture of the tangle. For example, $E_{n}$ may develop additional "fingers" or "branches" as it is mapped backwards. These fingers spread out into the phase space, creating additional intersections with the line of initial conditions. (In some cases, such fingers can be connected with the presence of an island chain inside the complex, such as the prominent period-5 chain in Fig. 1.) In general, a countable infinity of topological parameters is needed to completely describe the fingers, ${ }^{9,17-19}$ though we expect a finite number of parameters to suffice for the escape-time plot up to a given finite number of iterates. The homotopy formalism presented here can be generalized to incorporate these additional topological parameters, thereby predicting at least some of the strophe segments. We will address these issues in a future paper.

In future work, we will also study winding numbers, explaining the patterns shown in Fig. 2. In addition, we will apply our results to the ionization of hydrogen in parallel electric and magnetic fields.

\section{ACKNOWLEDGMENTS}

The authors would like to thank Professor Nahum Zobin for many useful discussions. This work was financially supported by the National Science Foundation.

\section{APPENDIX A: PROOF OF ALGORITHM 1}

We need only verify statements A and B in Algorithm 1. These are certainly true when all elements of the untangled basis are allowed in the expansion of $\ell_{N}$ (i.e., we do not omit the factors specified in step 1). This fact is evident by simply considering how a path is constructed from a reduced product of the basis paths shown in Fig. 5; at each occurrence of a $u_{n}$-factor, the path must cross under the hole and hence 
through $E_{n}$, thus creating an escape segment at the specified location (and with the specified direction). The $u_{n}$-factors are thus the key to determining the minimal set of escape segments. The $c_{n}$ basis elements $(n \leqslant D)$ create new $u_{n}$-factors via Eqs. (1b) and (3) and are thus themselves critical in determining the minimal set. However, the $e_{n}(n \geqslant 0)$ and $s_{n}$ $(-\infty<n<\infty)$ basis elements are "inert," mapping forward via Eqs. (1a) and (1d), never producing any $u_{n}$-factor. We thus lose nothing by eliminating them altogether from any expression which might contain them, as we have done in Eqs. (7)-(9). (One can verify that making these eliminations does not produce spurious cancellations of $c_{n}$ - or $u_{n}$-factors.)

\section{APPENDIX B: PROOF OF THEOREM 1}

Defining the two path-classes

$$
\begin{aligned}
& \gamma=u_{0} c_{1} u_{1}, \\
& \eta=u_{0}^{-1} F u_{\Delta}^{-1} F^{-1} u_{0},
\end{aligned}
$$

we have the following lemma (recalling that all $s_{n}$ and $e_{n}$ basis elements are omitted from our formulas).

Lemma: For any $N \geqslant N_{0}, \ell_{N}$ can be expressed as a product of elements in the set $S=\left(c_{1}, \ldots, c_{D} ; \square, \square\right.$, $\left.u_{2}, \ldots, u_{D}, \square, u_{D+2}, \ldots ; \gamma, \eta\right)$, assuming $D>1$; for $D$ $=1, S=\left(c_{1} ; \square, \square, \square, u_{3}, u_{4}, \ldots ; \gamma, \eta\right)$. (The symbol $\square$ emphasizes the absence of the classes $u_{0}, u_{1}, u_{\Delta}$. )

Proof of Lemma: It follows from the propagation formulas (1b), (1c), and (7) and the definitions of $n_{c}$ and $n_{u}$ that for $N \geqslant \max \left\{-n_{c}+1,-n_{u}, 0\right\}, \ell_{N}$ can be expressed as a product of the elements $\left(c_{1}, \ldots, c_{D} ; u_{0}, u_{1}, \ldots\right)$. Thus, for $N$ $\geqslant N_{0}, \ell_{N}$ can be expressed as a product of the elements $\left(c_{D+3}, \ldots, c_{2 D+2} ; u_{D+2}, u_{D+3}, \ldots\right)$. Since the elements $u_{D+2}, u_{D+3}, \ldots$ are in the set $S$, we need only verify that the elements $c_{D+3}, \ldots, c_{2 D+2}$ can be expressed as products of elements in $S$, a fact which follows from rewriting Eqs. (11d)-(11h) as

$$
\begin{aligned}
c_{D+3}= & \left(F^{-1} \gamma\right)\left(c_{2} u_{2}^{-1} c_{2}^{-1}\right)\left(\gamma^{-1} F\right), \\
c_{D+4}= & \left(F^{-1} \gamma c_{2} u_{2}\right)\left(c_{3} u_{3}^{-1} c_{3}^{-1}\right)\left(u_{2}^{-1} c_{2}^{-1} \gamma^{-1} F\right), \\
\vdots & \\
c_{D+n}= & \left(F^{-1} \gamma c_{2} u_{2} \ldots c_{n-2} u_{n-2}\right)\left(c_{n-1} u_{n-1}^{-1} c_{n-1}^{-1}\right) \\
& \times\left(u_{n-2}^{-1} c_{n-2}^{-1} \ldots u_{2}^{-1} c_{2}^{-1} \gamma^{-1} F\right) \\
\vdots & \\
c_{2 D+1}= & \left(F^{-1} \gamma c_{2} u_{2} \ldots c_{D-1} u_{D-1}\right)\left(c_{D} u_{D}^{-1} c_{D}^{-1}\right) \\
& \times\left(u_{D-1}^{-1} c_{D-1}^{-1} \ldots u_{2}^{-1} c_{2}^{-1} \gamma^{-1} F\right) \\
c_{2 D+2}= & \left(F^{-1} \gamma c_{2} u_{2} \ldots c_{D} u_{D}\right)\left(F^{-1} \eta F\right) \\
& \times\left(u_{D}^{-1} c_{D}^{-1} \ldots u_{2}^{-1} c_{2}^{-1} \gamma^{-1} F\right),
\end{aligned}
$$

for the case $D>1$. For $D=1$, Eq. (10d) yields $c_{4}$ $=c_{1}^{-1} \gamma c_{1}^{-1} \eta c_{1} \gamma^{-1} c_{1}$. This completes the proof of the lemma.

For $N \geqslant N_{0}$, we expand $\ell_{N}$ as a product of elements in $S$. By using Eqs. (B1) and (B2) to eliminate $\gamma$ and $\eta$, we obtain the expansion of $\ell_{N}$ in the untangled basis. It is easy to verify that when making these substitutions, there are no cancellations of any $u_{n}$-factors. (Here, we use the fact that powers of $\eta$, such as $\eta^{2}$, cannot occur in the expansion of $\ell_{N}$ since this would imply that $\mathcal{L}_{0}$ has a self-intersection.)

The theorem is now a trivial consequence of the representation of $\ell_{N}$ as a product of elements in the set $S$. Specifically, each occurrence of $\gamma$ in the product yields a single segment which escapes at $N-1$ iterates, corresponding to the $u_{1}$-factor of $\gamma$ in Eq. (B1), and a single segment which escapes at $N$ iterates, corresponding to the $u_{0}$-factor of $\gamma$. The form of Eq. (B1) also implies that the directions and relative positions of these two segments obey the Epistrophe Continuation Rule.

Similarly, Eq. (B2) implies that each occurrence of $\eta$ in the representation of $\ell_{N}$ yields a segment which escapes at $N-\Delta$ iterates and two segments which escape at $N$ iterates; the directions and relative positions of these segments obey the Epistrophe Start Rule. Since the basis elements $u_{0}, u_{1}$, and $u_{\Delta}$ occur in the expansion of $\ell_{N}$ only within the $\gamma$ - and $\eta$-factors, these two rules completely determine the minimal set of escape segments at $N$ iterates. $\mathcal{Q E D}$

${ }^{1}$ K. A. Mitchell, J. P. Handley, B. Tighe, J. B. Delos, and S. K. Knudson, Chaos 13, 880 (2003)

${ }^{2}$ D. W. Noid, S. K. Gray, and S. A. Rice, J. Chem. Phys. 84, 2649 (1986).

${ }^{3}$ J.-M. Petit and M. Henon, Icarus 66, 536 (1986).

${ }^{4}$ B. Eckhardt and C. Jung, J. Phys. A 19, L829 (1986).

${ }^{5}$ B. Eckhardt, J. Phys. A 20, 5971 (1987).

${ }^{6}$ C. Jung and H. J. Scholz, J. Phys. A 20, 3607 (1987).

${ }^{7}$ P. Gaspard and S. Rice, J. Chem. Phys. 90, 2225 (1989).

${ }^{8}$ P. Gaspard, Chaos, Scattering and Statistical Mechanics (Cambridge University Press, Cambridge, 1998).

${ }^{9}$ B. Rückerl and C. Jung, J. Phys. A 27, 55 (1994).

${ }^{10}$ B. Rückerl and C. Jung, J. Phys. A 27, 6741 (1994).

${ }^{11}$ C. Lipp and C. Jung, J. Phys. A 28, 6887 (1995).

${ }^{12}$ C. Jung, C. Lipp, and T. H. Seligman, Ann. Phys. (Leipzig) 275, 151 (1999).

${ }^{13}$ T. Bütikofer, C. Jung, and T. H. Seligman, Phys. Lett. A 265, 76 (2000).

${ }^{14}$ A. Tiyapan and C. Jaffé, J. Chem. Phys. 99, 2765 (1993).

${ }^{15}$ A. Tiyapan and C. Jaffé, J. Chem. Phys. 101, 10393 (1994).

${ }^{16}$ A. Tiyapan and C. Jaffé, J. Chem. Phys. 103, 5499 (1995).

${ }^{17}$ R. W. Easton, Trans. Am. Math. Soc. 294, 719 (1986).

${ }^{18}$ V. Rom-Kedar, Physica D 43, 229 (1990).

${ }^{19}$ V. Rom-Kedar, Nonlinearity 7, 441 (1994).

${ }^{20}$ A. Litvak-Hinenzon, M.Sc. thesis, Weizmann Institute, 1995

${ }^{21}$ S. Wiggins, Chaotic Transport in Dynamical Systems (Springer, New York, 1992).

${ }^{22}$ R. S. MacKay, J. D. Meiss, and I. C. Percival, Physica D 13, 55 (1984).

${ }^{23}$ M. Nakahara, Geometry, Topology, and Physics (IOP, Bristol, 1990).

${ }^{24}$ W. S. Massey, Algebraic Topology: An Introduction (Springer, New York, 1977).

${ }^{25}$ A. T. Fomenko, D. B. Fuchs, and V. L. Gutenmacher, Homotopic Topology (Akadémiai Kiadó, Budapest, 1986).

${ }^{26}$ In this paper, we do not require area-preservation and analyticity of $\mathcal{M}$, as in Assumption 1 of Paper I, but only that $\mathcal{M}$ be $C^{1}$.

${ }^{27}$ Topologically, the boundary of $A$ would be defined as its closure minus its interior and would therefore include the untangled branches of the stable and unstable manifolds. Here, when we say the boundary of $A$ we mean only the outer boundaries of the capture and escape zones as well as the $\mathrm{X}$ point.

${ }^{28}$ More formally, two (continuous) paths $\mathcal{A}_{1}, \mathcal{A}_{2}: I \rightarrow A^{*}$ (where $I=[0,1]$ ) are homotopic if there exists a continuous function $H: I \times I \rightarrow A^{*}$ such that $\forall t \in I, H(0, t)=\mathcal{A}_{1}(t), H(1, t)=\mathcal{A}_{2}(t)$ and $\forall \tau \in I, H(\tau, 0)=\mathbf{s}_{0}=\mathcal{A}_{1}(0)$ $=\mathcal{A}_{2}(0), H(\tau, 1)=\mathbf{s}_{1}=\mathcal{A}_{1}(1)=\mathcal{A}_{2}(1)$. The map $H$ is called a homotopy.

${ }^{29} \mathrm{~K}$. MacKenzie, Lie Groupoids and Lie Algebroids in Differential Geometry (Cambridge University Press, Cambridge, 1987).

${ }^{30}$ In practice, the line of initial conditions typically extends beyond the 
active region; here, we truncate it and consider only the piece inside $A$. In practice, the line of initial conditions may also pass through a hole $H_{n}$ or $\mathbf{z}_{\mathbf{X}}$; here we assume for simplicity that it does not. In Sec. IV B we consider an example in which $\mathcal{L}_{0}$ does intersect a hole $H_{n}$.

${ }^{31}$ Each end point can be shifted either forward or backward along $\partial A$. If an end point lies on $\mathcal{S}_{n}$, for some $n$, this choice does not affect the minimal set of escape segments. However, if an end point lies on $\mathcal{U}_{n}$, then that end of $\mathcal{L}_{0}$ terminates inside $E_{n}$, meaning that $\mathcal{L}_{0}$ ends in an escape segment. Depending upon the direction in which we shift this end point, we can either include or exclude this terminal segment from the minimal set. By convention, we shift the end point in the direction which includes the terminal segment. See the examples in Sec. IV.

${ }^{32}$ One may ask whether it is even possible to choose curves $\mathcal{L}_{0}^{\prime}, \mathcal{E}_{{ }_{-n_{1}}}^{\prime}$, and
$\mathcal{E}_{-n_{2}}^{\prime}$ satisfying the conditions of Question 2. That this is indeed possible is established by the following lemma, which is a simple corollary to a theorem of Turaev (Theorem 2 of Ref. 34). Lemma: Given a set of homotopy classes $a_{i} \in \Pi\left(A^{*}, \alpha\right), i=1, \ldots, m$, there exists a choice of representatives $\mathcal{A}_{i} \in a_{i}, i=1, \ldots, m$, such that for each $i$, the number of selfintersections of $\mathcal{A}_{i}$ is minimized and for each pair $i, j$, the number of pairwise-intersections between $\mathcal{A}_{i}$ and $\mathcal{A}_{j}$ is minimized.

${ }^{33}$ More generally, for a path $\mathcal{A}$ that does not intersect $\mathbf{z}_{\mathrm{X}}$ and that has a well-defined homotopy class in $\Pi\left(A^{*}, \alpha\right)$, the homotopy class of $\mathcal{A}$ has a (unique) finite reduced expansion in the basis.

${ }^{34}$ V. G. Turaev, Mat. Sb. 106, 566 (1978) [Math. USSR Sbornik 35, 229 (1979)]. 